\title{
Principal Component Prediction Model-based Analysis of Classification and Diagnosis of Sports Fatigue by Chinese Traditional Medicine
}

Q. LANG

Department of Sport medicine, Chengdu Sport University, Chengdu 610041, China

Lang: TCM Classification and Diagnosis of Sports Fatigue

\begin{abstract}
There has been a long-standing choice of traditional Chinese medicine for the treatment of sports fatigue in China. However, little research has been done on what factors drive exercise fatigue patients to use traditional Chinese medicine. Based on the representative samples in Beijing and Hefei, this study explored the possible factors for people to see a doctor in traditional Chinese medicine. The results showed that health information media exposure, self-assessment of health status, lifestyle, family income and working status were all related to traditional Chinese medicine treatment, but the impact of these factors on traditional Chinese medicine treatment was significantly different. This paper also discussed the tendency of functionalist use of traditional Chinese medicine and the cultural tradition of traditional Chinese medicine.
\end{abstract}

Key words: Traditional chinese medicine, western medicine, principal component prediction model, doctor-patient communication, media contact

In China, the traditional Chinese medical (TCM) system has coexisted for a long time. However, in the context of Western medicine becoming mainstream, comparison of TCM's advantages and disadvantages started and even resistance to TCM has surfaced ${ }^{[1,2]}$. In October 2012, a war on the diagnostic criteria of TCM caused concern on Weibo ${ }^{[3,4]}$. A doctor from the Jishuitan Burns Department in Beijing issued a challenge questioning the reliability of the blind diagnosis of $\mathrm{TCM}^{[5,6]}$. If TCM pregnancy prediction accuracy rate is more than $80 \%$, he would pay 50,000 bonuses and would not address the TCM as pseudoscience. Such challenges reflect the opposition to Chinese medicine ${ }^{[7,8]}$. At present, the research on TCM also regards the two as a zero-sum game. However, in the context of the dual medical system $^{[9]}$, which factors affect people's choice of TCM or Western medicine and there is lack of empirical data support ${ }^{[10]}$. Based on the theoretical framework and measurement indicators of the principal component prediction model (BMHSU), this paper empirically analysed the factors of TCM treatment selection. This model was proposed by Andersen and became the theoretical framework for the design of national and many regional health information questionnaires. In the context of a comparative study of TCM, this model mainly explores factors that influence people's choice of TCM, including social context characteristics, personal characteristics and results after TCM treatment. Personal characteristics can be derived from induced factors and possibilities. Factors, demand factors are considered (Table 1). The sample of this study was from a questionnaire survey on health information contact and use in Beijing and China's George Mason University in Beijing and Hefei in 2012. This survey is a trial survey of the nationwide survey of Chinese residents' health information in the future. This study is based on the principal component prediction model, which mainly discusses the predictive factors of TCM treatment and the variables from different parts of the questionnaire. The target population of this survey is residents of Beijing and Hefei. These two locations surveyed represented the political, economic and cultural centers of China and the level of secondary cities in China. The survey was conducted from October 16, 2012 to November 10, 2012. The survey used a multi-stage stratified random sampling method to extract a city 

TREATMENT CHOICES

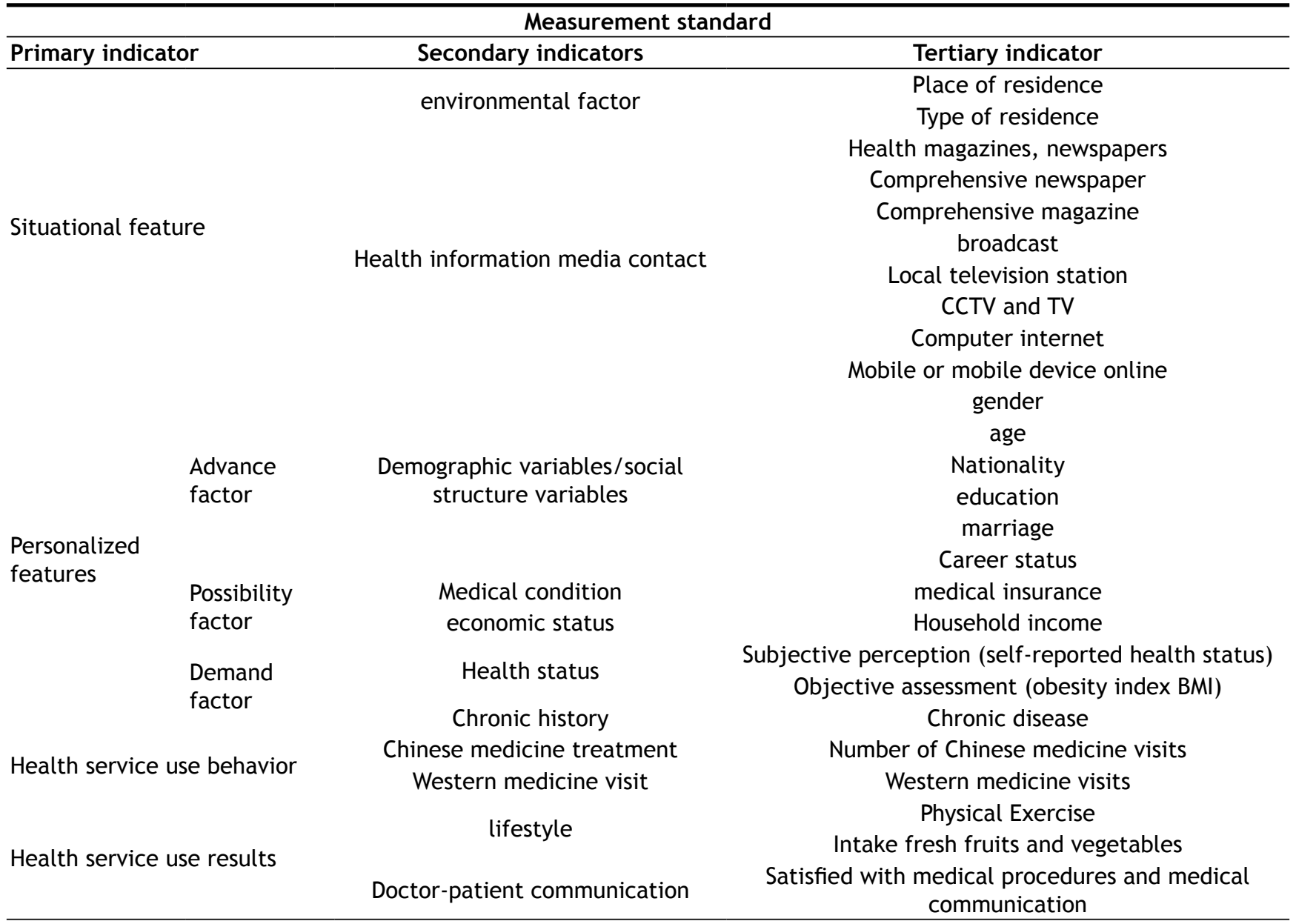

and a rural area in Beijing and Hefei as survey objects. According to the economy level, medium and poor, each district randomly draws 3 streets (townships); each street draws 2 neighbourhood communities (villages); each neighbourhood community draws 110 households; each household survey included 1 family member chosen based on whose birthday (date and month) is the closest to the survey date, and to ensure that the effective sample of each neighborhood community is not less than 100. According to the stratified method, the gender is both male and female, the age is 35 y (1/3) 36-60 y (2/3). The total of 110 households were randomly selected and 2,568 adult residents completed the survey. Table 2 shows that in this sample, the frequency of Western medicine visits is higher than that of to TCM practitioners. In the past 12 months, $45.90 \%$ of respondents have never seen a TCM practitioners, which is much higher than $8.65 \%$ of Western medicine. From the quality of doctorpatient communication, the respondents assessed their communication with the doctor as positive $(M=2.80 / 4)$. From the perspective of media health information, TV is the most important source, especially CCTV and David ( $\mathrm{M}=3.33 / 4)$, followed by local TV programs $(\mathrm{M}=3.15 / 4)$, and broadcast $(\mathrm{M}=2.49 / 4)$. There is also a high contact rate with professional health medical newspapers and magazines $(\mathrm{M}=2.42 / 4)$, but the mobile device health information contact rate is relatively low $(\mathrm{M}=1.84 / 4)$. Respondents reported a good self-reported health status with an average of 3.76 (5 measures). This result is consistent with the measurement of the obesity index (BMI), which shows that $65.51 \%$ of the respondents are in the normal range. On average, respondents reported that they had at least $20 \mathrm{~min}$ of vigorous physical exercise every month and they ate fresh fruit or vegetables every week. Sample population who reported a history of chronic disease is $55.4 \%$, with hypertension (17.51\%) and gastroenteritis (12.91 \%) being the two most common chronic diseases. From the demographic characteristics, the sample group has more males than female aged between 25 $59 \mathrm{y}$, Han people account for the vast majority (94.92 $\%)$ and most (65.26 \%) had an education level of high school and below. The geographical distribution of 
the sample population and the urban-rural distribution were relatively average (Table 2). The model was used to explore the relationship between the same group of predictors and the 2 types of doctors. Interestingly, when the frequency of TCM visits showed a significant positive correlation, most of the predictors were not continuous in the 2 models. Among all the predictors, only the doctor-patient communication was significant in both models. Therefore, the self-reports of the respondents indicated that there were more people who exchanged experiences with doctors in the past $12 \mathrm{mo}$. Compared with experienced people, there are more frequent visits. $(\beta=.12, p<.05$ and $\beta=.16, p<.01)$

Among the media exposure variables, the more contacts with professional health or medical print media, the more likely they were to seek TCM $(\beta=.10, p<.05)$. The frequency of local TV program exposure was significantly negatively correlated with the frequency of use of TCM treatment $(\beta=-.12, p<.05)$. The comprehensive document health information contact behavior was positively correlated with the frequency of Western medical treatment $(\beta=.10, p<.05)$ and the local TV program viewing behavior was negatively correlated with Western medicine treatment $(\beta=-.09, p=$. $06)$. Other types of health information media exposures were not significant for the frequency of TCM visits ${ }^{[3]}$. Different variables related to health status are also related to the relationship between TCM practitioners.

\section{TABLE 2: SAMPLE STATISTICAL DESCRIPTION}

\begin{tabular}{|c|c|c|c|}
\hline Type & Variable & Category & Proportion \\
\hline \multirow{10}{*}{ Dependent variable } & & 0 times & $45.90 \%$ \\
\hline & & $1-2$ times & $30.92 \%$ \\
\hline & Chinese medicine treatment (TCM) & 3-4 times & $9.73 \%$ \\
\hline & & $5-9$ times & $5.44 \%$ \\
\hline & & 10 times or more & $8.02 \%$ \\
\hline & Western medicine visit (WM) & 0 times & $8.65 \%$ \\
\hline & & $1-2$ times & $45.72 \%$ \\
\hline & & 3-4 times & $24.41 \%$ \\
\hline & & $5-9$ times & $9.56 \%$ \\
\hline & & 10 times or more & $9.56 \%$ \\
\hline \multirow{4}{*}{$\begin{array}{l}\text { Doctor-patient } \\
\text { relationship }\end{array}$} & Doctor-patient communication (5 options combined, $\alpha=.84$ ) & Never (1) - often (4) & $2.80(.67) \mathrm{a}$ \\
\hline & Health magazines, newspapers & & $2.42(1.06) \mathrm{a}$ \\
\hline & Comprehensive newspaper & & $2.28(1.07) \mathrm{a}$ \\
\hline & Comprehensive magazine & & $2.17(1.01) \mathrm{a}$ \\
\hline \multirow{6}{*}{$\begin{array}{l}\text { Health information } \\
\text { media contact }\end{array}$} & broadcast & Never (1) - often (4) & $2.49(1.09) \mathrm{a}$ \\
\hline & Local television station & sever (1)-orten (4) & $3.15(.95) \mathrm{a}$ \\
\hline & CCTV and TV & & $3.33(.88) \mathrm{a}$ \\
\hline & Computer internet & & $2.29(1.24) \mathrm{a}$ \\
\hline & Mobile or mobile device online & & $1.84(1.01) \mathrm{a}$ \\
\hline & Self-reported health status & $\begin{array}{l}\text { Very poor }(1) \text { - very } \\
\text { healthy (5) }\end{array}$ & $3.76(.79) \mathrm{a}$ \\
\hline Health status & Obesity Index (BMI) & $\begin{array}{l}\text { Overweight or too thin } \\
\text { normal }\end{array}$ & $\begin{array}{l}34.49 \% \\
65.51 \%\end{array}$ \\
\hline \multirow[t]{8}{*}{ living habit } & $\begin{array}{l}\text { Do more than } 20 \text { minutes of physical exercise } \\
\text { Intake fresh fruits and vegetables }\end{array}$ & $\begin{array}{l}\text { Never (1) - Everyday } \\
\text { (5) }\end{array}$ & $\begin{array}{l}3.12(1.49) \mathrm{a} \\
4.42(.96) \mathrm{a}\end{array}$ \\
\hline & hypertension & & $17.51 \% / 82.49 \%$ \\
\hline & Gastroenteritis & whether & $12.91 \% / 87.09 \%$ \\
\hline & Diabetes/high blood sugar & & $5.98 \% / 94.02 \%$ \\
\hline & Rheumatoid Arthritis & & $5.23 \% / 94.77 \%$ \\
\hline & Disc disease & & $9.13 \% / 90.87 \%$ \\
\hline & Chronic obstructive pulmonary disease & & $1.06 \% / 98.94 \%$ \\
\hline & Ischemic heart disease & & $2.24 \% / 97.76 \%$ \\
\hline \multirow{5}{*}{$\begin{array}{l}\text { Chronic disease } \\
\text { types }\end{array}$} & Cerebrovascular disease & whether & $1.77 \% / 98.23 \%$ \\
\hline & Gallstones, cholecystitis & & $3.86 \% / 96.14 \%$ \\
\hline & Peptic ulcer & & $3.27 \% / 96.73 \%$ \\
\hline & Other chronic diseases & & $2.99 \% / 97.01 \%$ \\
\hline & No history of chronic disease & & $55.41 \% / 44.59 \%$ \\
\hline
\end{tabular}




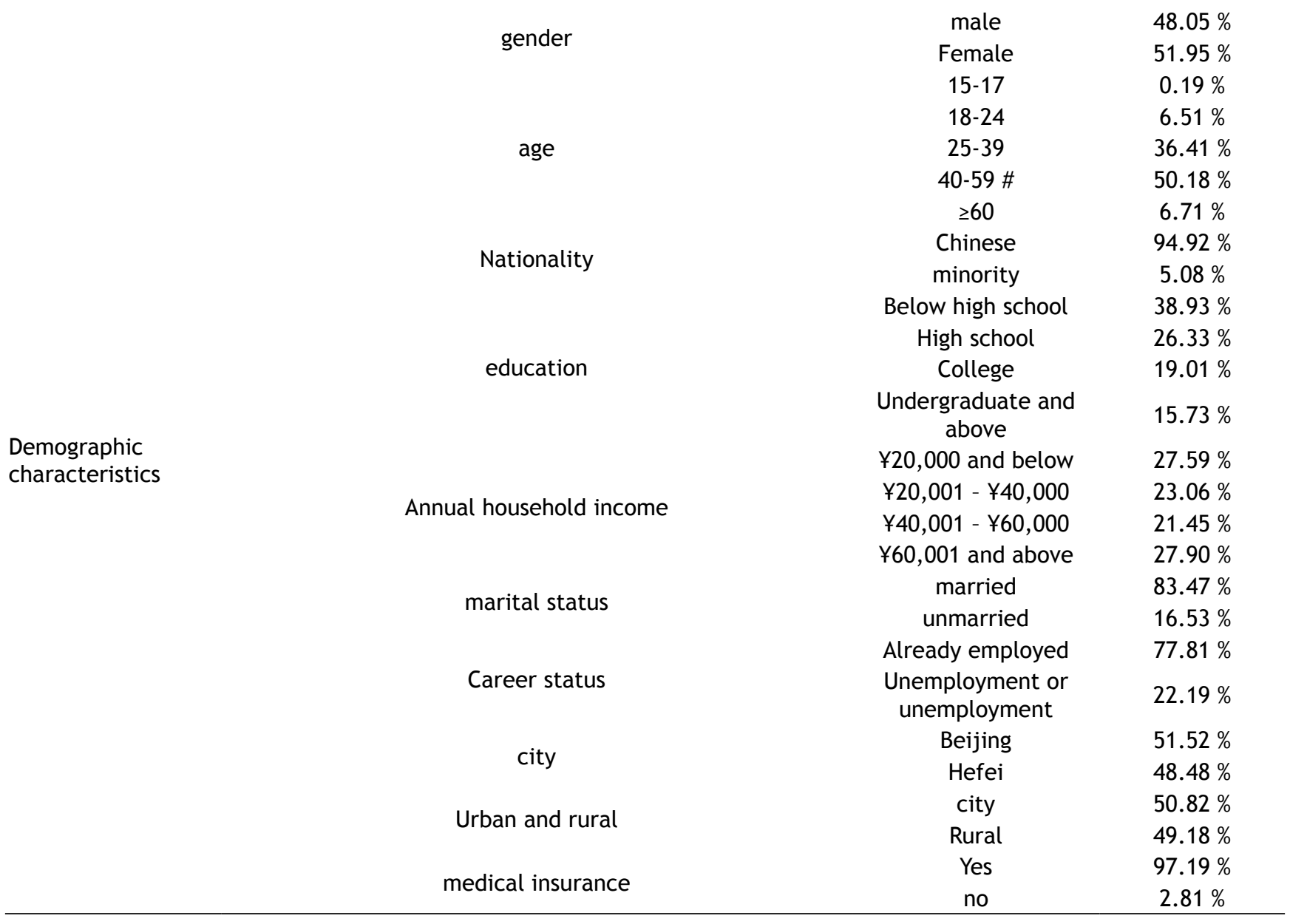

For TCM practitioners, self-reported health status and objective health status (BMI) are not significant factors in the treatment of TCM. Those who took more fruits and vegetables were more likely to visit TCM practitioner $(\beta=.11, p<.01)$, but the frequency of exercise and Chinese medicine was not significant $(\beta=.02, p=.36)$. Among the 11 common chronic diseases, those with a history of intervertebral disc disease $(\beta=.31, p<.01)$ and other chronic diseases $(\beta=.56, p<.01)$ were more likely to visit TCM. For Western medicine, the self-reported health status is significantly negatively correlated with the frequency of Western medical treatment, that is, the more likely it is that the respondents who are healthy are less likely to visit Western medicine $(\beta=$ $.16, p<.001)$. Often physical exercise $(\beta=.07, p<.01)$, hypertenson $(\beta=.33, p<.001)$ and ischemic heart disease $(\beta=.55, p<.01)$ the respondents are more likely to choose Western medicine. China's sports fatigue has long been in the environment of coexistence of TCM and the coexistence of 2 medical systems can be traced back to the Ming Dynasty. In today's modern society of China, both medical systems are valued and licensed. The core concept of TCM emphasizes the use of gentle methods to reshape the balance of the body, which is also advocated by most Chinese. Therefore, China is an important environment for studying people's medical choices. This study is based on a principal component predictive model (Andersen, 2008) to explore a range of influencing factors that predict Chinese TCM treatment for exercise-induced fatigue in China, such as doctor-patient communication, media health information exposure, health-related factors and demographic characteristics. The study found that TCM practitioners with exercise-induced fatigue showed more a combination of complementarity rather than competition $(\beta=.24, p<.001$ and $\beta=.21, p<.001$; Table 2). Moreover, the study also found some of the same or different factors associated with the two types of visits. These findings have some implications for both the theory and practice of different medical services. The conclusions indicate that media exposure has different effects on the behavior of TCM treatment. Respondents who are exposed to a large number of professional medical print media are more inclined to TCM. The possible explanation is that professional health medical print media is relatively more reliable, and is also more likely to transmit scientific knowledge of TCM. The local TV contact frequency is inversely 
related to the TCM. According to previous research, television is more of a medium for entertainment than for disseminating knowledge, and local TV is better able to target advertisements to target audiences than national TV stations. Because of this, many local TV stations are filled with various medical advertisements, such as pharmaceutical commercial advertisements, TV shopping, health programs and many are even considered to be counterfeit and shoddy products. Some studies on the effectiveness of medical advertisements showed that many TCM advertisements have exaggerated the treatment effect, which is more likely to cause resentment from the viewers. These could be the reasons why people who have access to local TV health information are less likely to choose TCM. The health information contact of comprehensive newspapers is positively related to Western medical treatment, which may be related to the mainstream propaganda and scientific label of Western medicine. In TCM, regular physical exercise is considered to effectively strengthen the immune system and reduce the risk of disease. Respondents who have a healthy lifestyle and emphasize regular physical exercise are more likely to accept Western medicine concepts and are more likely to have Western medicine. Although the contrasting interesting findings (exercise and dieting) of this lifestyle choice are not mutually exclusive, they provide important inspirations in lifestyle and medical decision-making. The experimental results also showed that effective and satisfactory communication between doctors and patients is related to the number of visits to exercise-induced fatigue, which is applicable to TCM. The survey was conducted only in Beijing and Hefei. These two locations are actually larger eastern cities, which are more developed than inland areas, so future national representative surveys may expand the universality of this study. In summary, for medical practitioners, understanding the factors that influence the medical decision-making of exercise-induced fatigue can help them to meet the needs of exercisestimulated people to the greatest extent and achieve better medical outcomes.

\section{REFERENCES}

1. Sepulveda E, Franco JG, Trzepacz PT, Gaviria AM, Eva
V, Palma J, et al. Performance of the delirium rating scalerevised-98 against different delirium diagnostic criteria in a population with a high prevalence of dementia. Psychosomatics 2015;56(5):530-41.

2. Michiels JJ, Berneman Z, Schroyens W, De Raeve H. Changing concepts of diagnostic criteria of myeloproliferative disorders and the molecular etiology and classification of myeloproliferative neoplasms: from dameshek 1950 to vainchenker 2005 and beyond. Acta Haematologica 2015;133(1):36-51.

3. Wilson JE, Niu K, Nicolson SE, Levine SZ, Heckers S. The diagnostic criteria and structure of catatonia. Schizophr Res 2015;164(1-3):256-62.

4. Carrillo-De-La-Pe AMT, Tri?Anes Y, González-Villar A, Romero-Yuste S, Gómez-Perretta C, Arias ML, et al. Convergence between the 1990 and 2010 acr diagnostic criteria and validation of the spanish version of the fibromyalgia survey questionnaire (fsq). Rheumatol Int 2015;35(1):141-51.

5. Bower JH, Maraganore DM, Mcdonnell SK, Rocca WA. Influence of strict, intermediate, and broad diagnostic criteria on the age- and sex-specific incidence of parkinson's disease. Movement Disorders 2015;15(5):819-25.

6. Dumesic DA, Oberfield SE, Stenervictorin E, Marshall JC, Laven JS, Legro RS. Scientific statement on the diagnostic criteria, epidemiology, pathophysiology, and molecular genetics of polycystic ovary syndrome. Endocr Rev 2015;36(5):487-525.

7. Lethielleux G, Amar L, Raynaud A, Plouin PF, Steichen O. Influence of diagnostic criteria on the interpretation of adrenal vein sampling. Hypertension 2015;65(4):849-54.

8. Valent P, Sotlar K, Blatt K, Hartmann K, Reiter A, Sadovnik $\mathrm{I}$, et al. Proposed diagnostic criteria and classification of basophilic leukemias and related disorders. Leukemia 2017;31(4):788-97.

9. Hauptmann S, Friedrich K, Redline R, Avril S. Ovarian borderline tumors in the 2014 who classification: evolving concepts and diagnostic criteria. Virchows Arch, 2017;470(2):125-42.

10. Wilson D, Chatterjee F, Farmer SF, Rudge P, Mccarron $\mathrm{MO}$, Cowley $\mathrm{P}$, et al. Infratentorial superficial siderosis: classification, diagnostic criteria, and rational investigation pathway. Annals of Neurology 2017;81(3):333-43.

This is an open access article distributed under the terms of the Creative Commons Attribution-NonCommercial-ShareAlike 3.0 License, which allows others to remix, tweak, and build upon the work non-commercially, as long as the author is credited and the new creations are licensed under the identical terms

This article was originally published in a special issue: Special issue on "Animal Models \& Experimental Medicine"

Indian J Pharm Sci 2020:82(1)spl issue4;40-44 